\title{
Integration of function, aesthetics and patients' personal preferences in the prosthetic treatment planning
}

\author{
Georgi lliev \\ Medical university of Sophia, Faculty of Dental Medicine, Department for Prosthetic dentistry, Sofia, Bulgaria
}

\begin{abstract}
SUMMARY
Introduction Optimal aesthetic results require suitable smile design that fulfils patient's expectations. Psychological importance of teeth appearance is clear and often discussed in relation to the success of prosthetic treatment. The objective of this article was to present methodology for creating customized smile design using the Visagismile concept and evaluate aesthetic satisfaction with prosthetic treatment.

Case report A 52-year-old female patient required complete esthetical dental mouth reconstruction. Digital planning software (Visagismile) provided dentists and technicians a 2D preview of the final design that relates facial perception and personality of the patient. The latest innovation of used software in addition to documents that dentists send for the Visagismile application is that they need to send an intraoral 3D scan of the patients mouth (with any scanner) and define the length and position of incisal edges of the central incisors. This information as a STL file is sent to new Visagismile/REBEL center that is actually a digital lab, that converts 2D designs created by the Visagismile concept into 3D and create a digital wax up immediately.

Conclusions Visagismile concept supports both dentist and patient in deciding the aesthetics of the prosthetic restoration. The total coefficient of the assessment of patients for the final aesthetic result was highly satisfactory. Using the proposed methodology, a smile design corresponding to individual facial features, temperament and personal preferences of the patient can be reproduced.
\end{abstract}

Keywords: personalized digital design; esthetic treatment; prosthetic treatment

\section{INTRODUCTION}

Optimal aesthetic results require suitable smile design that satisfies patient's expectations largely influenced by psychological peculiarities of character, social status and education $[1,2,3]$. Psychological importance of good appearance of teeth is clear and often discussed in relation to the success of prosthetic treatment [4]. Detailed functional analysis of dentition using provisional restorations to change or adapt the smile design is also needed $[5,6]$. To build the design of future prosthetic work, contemporary dental medicine uses digital technology in order to meet maximum requirements $[7,8]$. Papasotiriou et al. studied the effectiveness of computer visualization and applicability in clinical practice. They examined two groups. Patients from the first group consulted by computer visualization showed higher satisfaction with the treatment $(93.75 \%)$ compared to conventionally consulted (83.3\%) [9].

The objective of this article was to present the methodology for creating customized smiles designs using the Visagismile concept and evaluates aesthetic satisfaction after prosthetic treatment.

\section{CASE REPORT}

A woman of age 52 required esthetic prosthetic reconstruction of her teeth. After the full-face picture of the patient with half smile was taken it was revealed that her smile doesn't show any teeth (very short teeth). However, a stretched smile showed incisal edges of the upper incisors. A digital planning software Visagismile provided dentists and technicians a 2D preview of the final design that related facial perception and personality of the patient to the smile design, using thousands of algorithms for the combination of incisal silhouette, tooth axis, dominance of the centrals and combination of individual tooth shapes $[10,11]$. The latest innovation of the software is, in addition to documents that dentists used to send for the Visagismile application, they only need to send intraoral 3D scan of patients mouth (with any scanner), define the length and position of incisal edges of central incisors and send this information as a STL file to new Visagismile/ REBEL center. The new REBEL system is actually a digital lab, that converts 2D designs created by the Visagismile concept in to 3D, and creates digital wax up immediately. Current esthetic software programs can be powerful tools in assessing and modifying the design of deficient smiles [12, 13]. In this case, impressions from the upper and lower jaw with standard metal trays and impression material were taken. Plaster-working models were made. They were placed in the articulator and a wax modeling of the upper front teeth was produced in accordance with the parameters set by the Visagismile design. Silicone keys with high hardness were made on wax prototypes. In the silicon index a Bis-acril self-cured composite was placed 


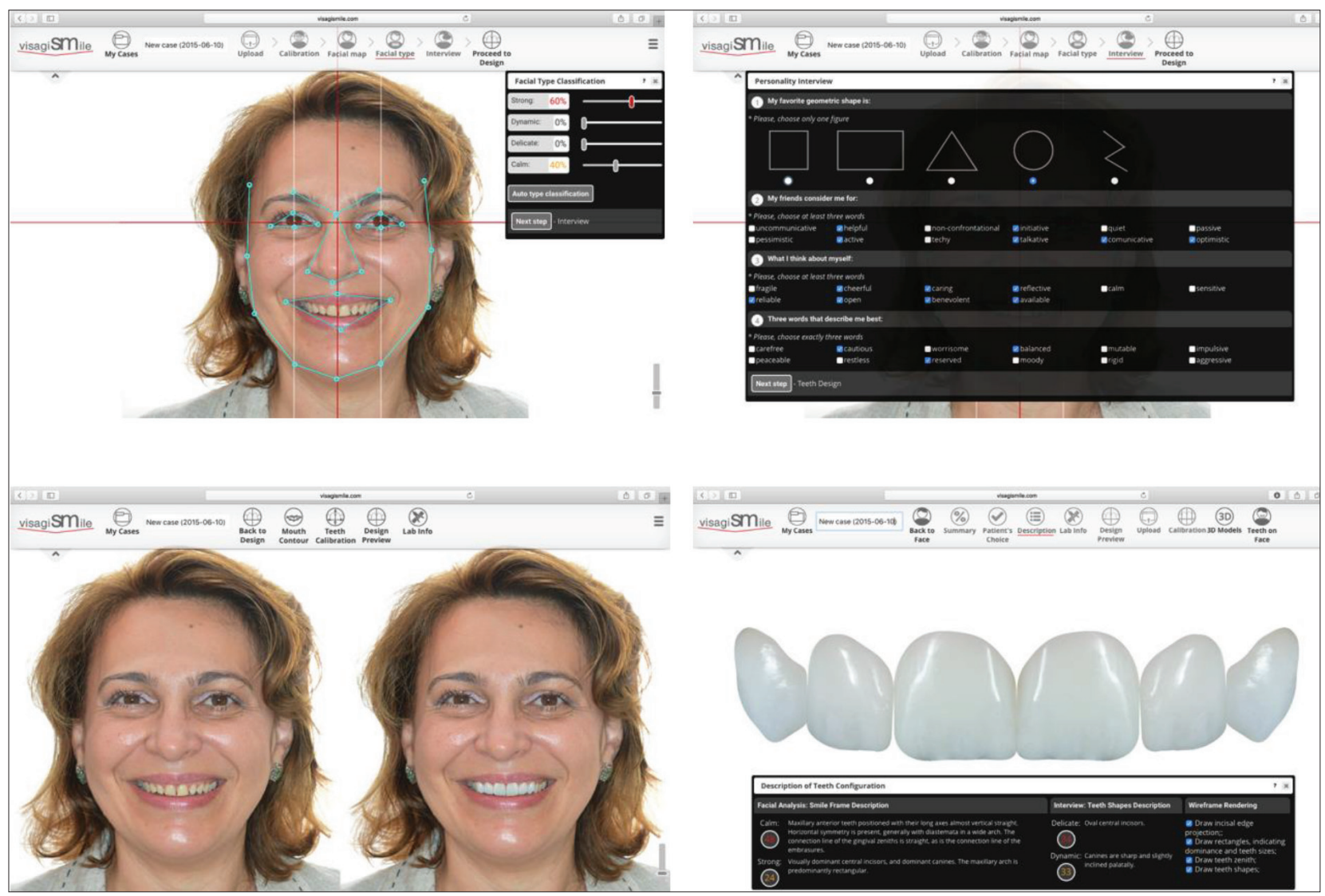

Figure 1. Workflow of Visagismile software, face reading, interview, design preview Slika 1. Vorkflov softver Visagismile, čitanje lica, intervju, pregled dizajna
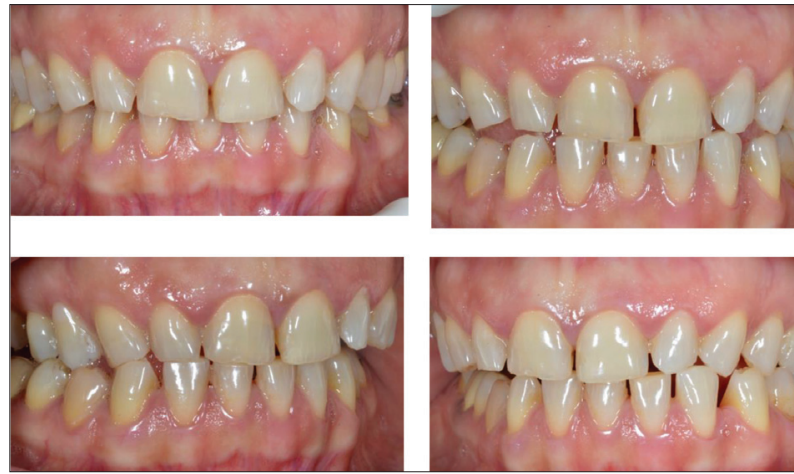

Figure 2. Initial situation of upper and lower teeth: central occlusion, protrusion, right and left laterotrusion

Slika 2. Inicijalna situacija gornjih i donjih zuba: centralna okluzija, protruzioni položaj, desni i levi laterotruzioni položaj
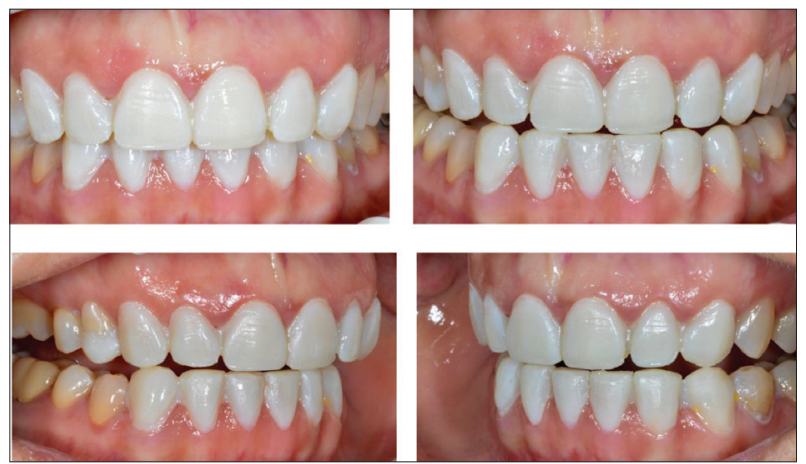

Figure 4. Mock up of upper and lower teeth: central occlusion, protrusion, right and left laterotrusion

Slika 4. Proba gornjih i donjih zuba: centralna okluzija, protruzioni položaj, desni i levi laterotruzioni položaj
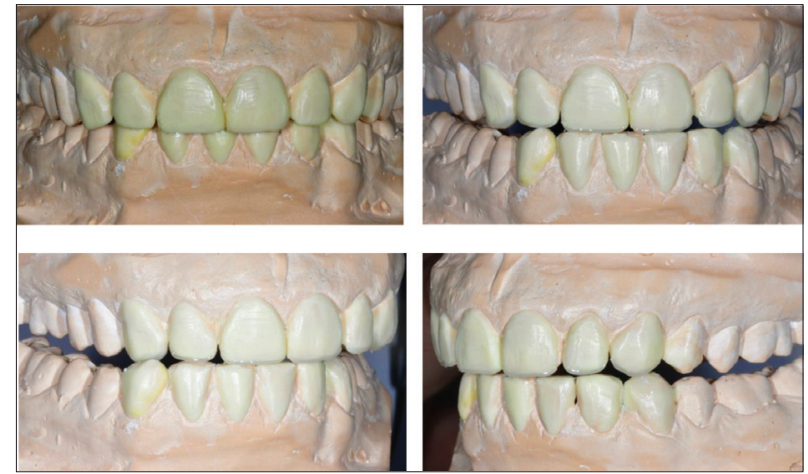

Figure 3. Wax-up of upper and lower teeth: central occlusion, protrusion, right and left laterotrusion

Slika 3. Voštani model gornjeg i donjeg zubnog niza: centralna okluzija, protruzioni položaj, desni i levi laterotruzioni položaj
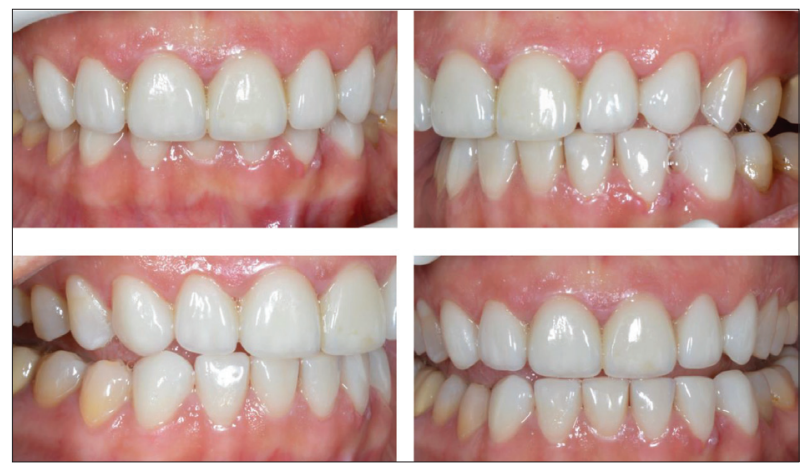

Figure 5. Final restorations of upper and lower teeth: central occlusion, protrusion, right and left laterotrusion.

Slika 5. Završna restauracija gornjih i donjih zuba: centralna okluzija, protruzioni položaj, desni i levi laterotruzioni položaj 


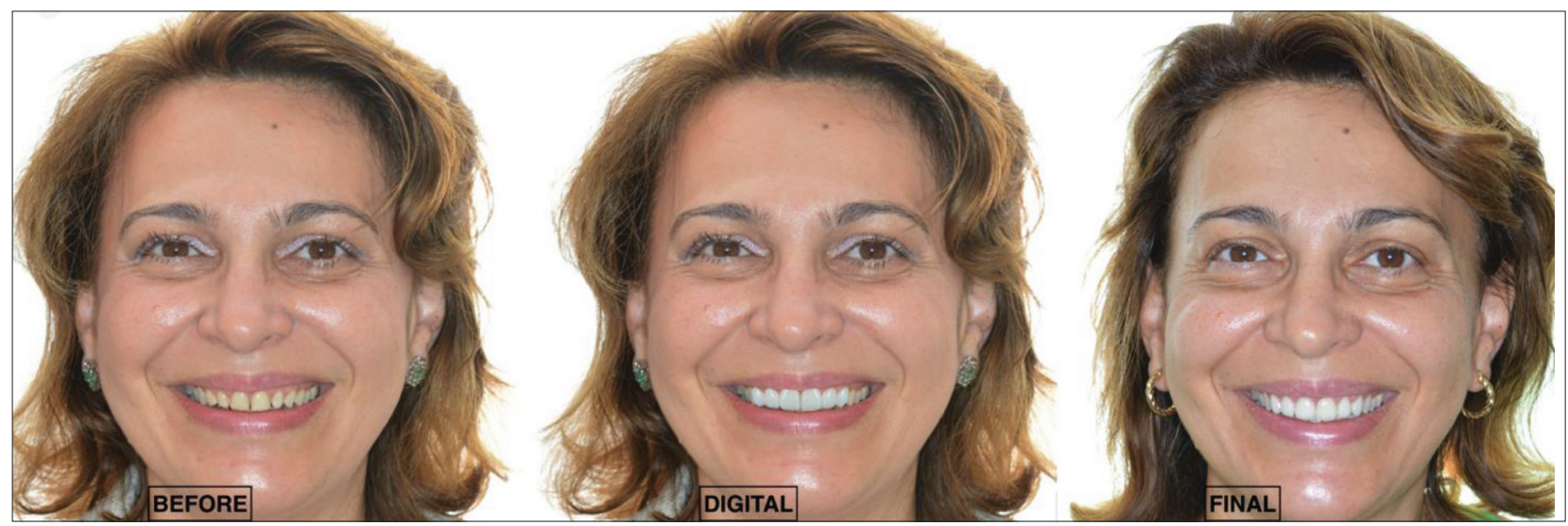

Figure 6. Final restorations of upper and lower teeth: before,digital planing, final restorations. Slika 6. Završne restauracije gornjeg i donjeg zuba: ranije, digitalno planiranje, završne restauracije

and the design was recreated in the mouth in the form of a composite mask over the patient's natural teeth. After the person's consent, the treatment was completed with definitive prosthetic work [5]. The main advantage of the presented clinical case was minimally invasive treatment. The old composite restorations needed to be replaced because of existing microleakage. The canting of the occlusal plane and the abrasion of incisal edges were consistent with functional problems. There was also a diastema and spacing between the front teeth. At the lower jaw, there was one missing premolar with space that distributed among other teeth. By adding volume on the buccal surface and incisal edge of the teeth on the upper and lower jaw, positioning them more vestibularly, we could expand the arch and be minimally invasive and totally additive in this particular case. By achieving optimal teeth proportions, we restored normal occlusion in protrusion and laterotrusion, and removed functional disorders. The treatment plan for restoring upper teeth included six lithium-disilicate veneers on the front six teeth, partial veneers on first premolars, and lithium-disilicate overlays on molars. Follow-up of the case lasted three years and the patient had no complications. There were no ceramic chippings or debonding. Function and aesthetics were as preserved as on the day one.

The patient was asked to complete a questionnaire (orofacial aesthetic scale of Larsson [12]), which evaluates the aesthetic result of individual applications design. Results showed high degree of satisfaction regarding the teeth shade, gum appearance and changes in overall appearance of her smile and teeth.

\section{CONCLUSION}

Visagismile concept supports dentists and patients in deciding about aesthetics of prosthetic restoration. The total coefficient representing the final aesthetic result of the prosthetic treatment was satisfactory. Using the proposed methodology, smile design corresponding to individual facial features, the temperament, and personal preferences of the patient can be achieved.

\section{REFERENCES}

1. Paolucci B, Calamita M, Coachman Ch, Gurel G, Shaider A, Hallawell P. Visagism: The art of dental composition. Quintess Dent Technol. 2012; 35:187-201.

2. Pauwels R, Jacobs R, Singer SR, Mupparapu M. CBCT-based bone quality assessment: are Hounsfield units applicable? Dentomaxillofac Radiol. 2015; 44:20140238. [DOI: 10.1259/dmfr.20140238] [PMID: 25315442]

3. Roumanas ED. The social solution - denture esthetics, phonetics, and function. J Prosthodont. 2009; 18:112-5. [DOI: 10.1111/j.1532849X.2009.00440.x] [PMID: 19254300]

4. Davis LG, Ashworth PD, Springs LS. Psychological effects of aesthetic dental treatment. J Dent. 1998; 26:547-54. [DOI: 10.1016/ S0300-5712(97)00031-6] [PMID: 9754742]

5. Gürel G, Bichacho N. Permanent diagnostic provisional restorations for predictable results when redesigning smiles. Pract Proced Aesthet Dent. 2006; 8:281-6. [PMID: 16903532]

6. Zaccaria M, Squadrito N. Photographic-assisted prosthetic design technique for the anterior teeth. Int J Esthet Dent. 2015 Spring; 10(1):48-67. [PMID: 25625127]

7. Peixoto S, Freitas de Almedia FN, Ferreira D, Mesquita M, Negreiros W. Digitized study of the correlation between the face and tooth shapes in young adult individuals. Braz J Oral Sci. 2007; 6:1383-6.

8. Zimmermann M, Mehl A. Virtual smile design systems: a current review. Int J Comput Dent. 2015; 18:303-17. [PMID: 26734665]

9. Papasotiriou OS, Nathanson D, Goldstein RE. Computer imaging versus conventional esthetic consultation: a prospective clinical study. J Esthet Restor Dent. 2000; 12:72-7. [PMID: 11326506]

10. Iliev, G. Personalized Digital Smile Design for Predictable Aesthetic Results. Balk J Dent Med. 2016; 20:172-7.

11. Yankov B, lliev G, Filtchev D, Gurel G, Paolucci B, Schayder A, et al. Software Application for Smile Design Automation Using the Visagism Theory. Proceedings of the 17th International Conference on Computer Systems and Technologies, CompSysTech'16, June 23-24, Palermo, Italy, ACM International Conference Proceeding Series, Vol. 1164, ACM Inc., N.Y. USA, p. 237-44. [DOI: 10.1145/2983468.2983521]

12. Larsson P, John T, Nilner K, Bondemark L, List T. Development of an Orofacial Esthetic Scale in Prosthodontic Patients. Int J Prosthodont. 2010; 23:249-56.

13. Feraru M, Bichacho N, Muzella V. Individualizing a smile makeover. Current strategies for predictable results. J Cosmet Dent. 2016; 32:109-19.

Received: 04.09.2017 • Accepted: 28.11.2017 


\title{
Integracija funkcije, estetike i pacijentovih ličnih želja u planiranju protetskog tretmana
}

\author{
Georgi Iliev \\ Medicinski univerzitet, Fakultet za dentalnu medicinu, Odeljenje za protetsku dentalnu medicinu, Sofija, Bugarska
}

\begin{abstract}
KRATAK SADRŽAJ
Uvod Optimalni estetski rezultati zahtevaju odgovarajući dizajn osmeha koji ispunjava očekivanja pacijenta. Psihološki značaj dobrog izgleda zuba je jasan i često u vezi sa uspehom protetskog tretmana.

Cilj ovog rada je bio da se predstavi metodologija za kreiranje prilagođenog dizajna osmeha koristeći koncept Visagismile i da se proceni zadovoljstvo pacijenta posle protetske terapije.

Prikaz slučaja Žena od 52 godine je zahtevala estetsku terapiju zuba. Korišćen je program digitalnog planiranja Visagismile, koji stomatolozima i zubnim tehničarima pruža 2D pregled finalnog dizajna osmeha, a u vezi sa percepcijom lica i ličnosti pacijenta. Najnovija inovacija ovog softvera je u tome što treba poslati samo intraoralni 3D snimak osmeha pacijenata (sa bilo kojim skenerom) uz definisanje dužine i pozicije incizalnih ivica centralnih sekutića kao STL datoteku novom Visagismile / REBEL centru, gde ova digitalna laboratorija odmah formira digitalni vosak. Postojeći estetski softverski programi su moćni alati u proceni i modifikaciji dizajna neadekvatnih osmeha.

Zaključak Visagismile koncept olakšava i pomaže stomatologu i pacijentu u planiranju estetike protetske nadoknade. Ukupan koeficijent procene pacijenata o konačnom estetskom rezultatu protetskog rada bio je veoma zadovoljavajući. Predloženi protetski metod protetike može reprodukovati dizajn osmeha koji odgovara pojedinačnim osobinama lica, temperamentu i ličnim željama pacijenta. Ključne reči: personalizovani digitalni dizajn; estetski tretman; protetski tretman
\end{abstract}

\section{UVOD}

Optimalni estetski rezultati podrazumevaju odgovarajući dizajn osmeha koji ispunjava očekivanja pacijenta [1,2], na koja u velikoj meri utiču psihološke osobenosti nekog karaktera, društveni status i obrazovanje [3]. Psihološki značaj dobrog izgleda zuba je jasan, i često zavisi od uspeha protetskog tretmana [4].

Često je potrebna i detaljna funkcionalna analiza denticije, kojom se uspostavljaju privremene promene ili prilagođava sam dizajn osmeha $[5,6]$. Za dizajniranje budućeg protetskog rada savremena stomatologija koristi digitalne tehnologije u pokušaju da ispuni maksimalne zahteve pacijenata [7, 8, 9].

Papasotiriou, Nathanson i Goldstein [10] proučavali su efikasnost računarske vizualizacije i primenljivosti u kliničkoj praksi. Ispitivane su dve grupe. Pacijenti iz prve grupe su ispitivani pomoću računarske vizualizacije i bili su zadovoljniji - 93,75\%, $\mathrm{u}$ poređenju sa pacijentima sa konvencionalnim konsultacija$\mathrm{ma}-83,3 \%$.

Cilj ovog rada je bio da se predstavi metodologija za kreiranje prilagođenog dizajna osmeha koristeći koncept Visagismile i da se proceni zadovoljstvo pacijenta estetskim rešenjem posle protetske terapije.

\section{PRIKAZ SLUČAJA}

Žena od 52 godine je zahtevala estetski tretman zuba. Pripremljena je fotografija lica pacijenta sa blagim osmehom. Ukoliko se na fotografiji ne vide zubi (pacijenti sa vrlo kratkim zubima), pacijent se slika sa širokim osmehom koji omogućava prikazivanje incizalnih ivica gornjih sekutića. Program digitalnog planiranja koji je korišćen u vreme tretmana bio je Visagismile [11], koji stomatolozima i zubnim tehničarima pruža 2D pregled finalnog dizajna osmeha, a u vezi je sa percepcijom lica i ličnosti pacijenta, jer omogućava rad sa hiljadama algoritama za kombinaciju incizalne siluete, ose zuba, dominacije centralnih inciziva i kombinacije pojedinačnih oblika zuba. Najnovija inovacija ovog softvera je u tome da je, pored dokumenata koje su stomatolozi koristili za slanje Visagismile, neophodno samo poslati intraoralni 3D snimak usta pacijenata (sa bilo kojim skenerom) i definisati dužinu i poziciju incizalnih ivica centralnih sekutića. Ovu informaciju treba poslalati kao STL datoteku novom Visagismile / REBEL centru. Novi REBEL sistem je digitalna laboratorija koja odmah formira digitalni vosak. Može zvučati komplikovano, ali je to najjednostavniji način da se postigne jedan od najboljih 3D digitalnih voskova. Postojeći estetski softverski programi mogu biti moćni alati u proceni i modifikaciji dizajna osmeha $[12,13,14]$.

Za konkretni slučaj su uzeti otisci gornje i donje vilice sa standardnim metalnim kašikama i materijalom za otiske. Izrađeni su gipsani modeli i fiksirani u artikulatoru, a modelovanje u vosku gornjeg fronta zuba izrađeno je u skladu sa parametrima postavljenim Visagismile dizajnom. Silikonski trejevi visoke tvrdoće napravljeni su na voštanim prototipovima. U silikonski trej je postavljen bis-akrilni samovezujući kompozit, a nakon toga je trej postavljen u usta pacijenta preko prirodnh zuba i na taj način formirana kompozitna maska u vidu novodizajniranog osmeha. Posle saglasnosti pacijenta terapija je završena definitivnim protetskim radom [15]. Glavna prednost u predstavljenom kliničkom slučaju je u tome što je ovde predstavljena minimalno invazivna procedura za zubne strukture. Stare kompozitne restauracije je bilo neophodno zameniti zbog postojećih mikropukotina. Zaravnjene okluzalne površine zuba i abrazija incizalnih ivica su takođe predstavljale postojeće funkcionalne probleme. Postojali su dijasteme i razmaci između prednjih zuba. U donjoj vilici nedostajao je jedan premolar, te je taj prostor morao biti „podeljen“ među drugim zubima. Dodavanjem materijala na bukalnoj površini i incizalnoj ivici zuba gornje i donje vilice i pozicioniranjem vestibularno proširen je luk i obezbeđen minimalno invazivni tretman i potpuno odgovarajući u ovom konkretnom slučaju. Postizanjem optimalnih proporcija zuba normalna okluzija se može vraćati u protruzioni i laterotruzioni smer i time ukloniti funkcionalne poremećaje. Plan terapije za restauraciju gornjih 
zuba obuhvatao je šest litijum-disilikatnih venira na prednjih šest zuba, poluvenire na prvim premolama i litijum-silikatne overleje na molarima. Praćenje slučaja je trajalo tri godine, a pacijent nije imao komplikacija. Nije bilo odlamanja keramike niti rascementiranja. Funkcija i estetika su bili očuvani kao i prvog dana.

Od pacijenta je zatraženo da popuni upitnik (orofacijalnu estetsku skalu Larsson [13], koja procenjuje estetski rezultat dizajna pojedinačnih slučajeva. Sumirani rezultati iz odgovora pacijenta na pitanja pokazuju koeficijent koji ukazuje na zadovoljstvo pacijenta u pogledu boje zuba, vidljivosti desni i ukupnog izgleda osobe u odnosu na promene koje se javljaju u pogledu osmeha i zuba.

\section{ZAKLJUČAK}

Visagismile koncept pomaže stomatologu i pacijentu u planiranju estetike protetske nadoknade. Ukupan koeficijent procene pacijenata o konačnom estetskom rezultatu protetskog rada bio je veoma zadovoljavajući. Predloženi protetski metod protetike može reprodukovati dizajn osmeha koji odgovara pojedinačnim osobinama lica, temperamentu i ličnim željama pacijenta. 